\title{
Analyzing the Fermi Bubbles with DArk Matter Particle Explorer
}

\section{Zhao-Qiang Shen, ${ }^{a, *}$ Kai-Kai Duan, ${ }^{a}$ Zun-Lei Xu, ${ }^{a}$ Xiang Li ${ }^{a}$ and Qiang Yuan ${ }^{a, b}$ on behalf of the DAMPE ${ }^{\dagger}$ Collaboration}

(a complete list of authors can be found at the end of the proceedings)

${ }^{a}$ Key Laboratory of Dark Matter and Space Astronomy, Purple Mountain Observatory, Chinese Academy of Sciences, Nanjing 210033, China

${ }^{b}$ School of Astronomy and Space Science, University of Science and Technology of China, Hefei 230026, China

E-mail: zqshen@pmo.ac.cn

The Fermi bubbles are two large structures above and below the Galactic Plane. They are first discovered by Fermi-LAT and thought to be related to the jet or the wind from the Galactic center. The DArk Matter Particle Explorer (DAMPE) is a space-borne high energy particle telescope aiming at measuring cosmic rays and photons in a broad energy range. In this work, we use 4.8 years of DAMPE photon data to search for the emission from the Fermi Bubbles. We calculate the TS values of the lobes and the significance of its curved spectrum. The obtained spectral parameters are then compared with those from the Fermi-LAT. We also search for the emission from the cocoon in the southeast part of lobes. Since the Galactic diffuse emission (GDE) model is a major source of systematic uncertainty, we also switch to the GDE models calculated with Galprop and evaluate the influence.

$37^{\text {th }}$ International Cosmic Ray Conference (ICRC 2021)

July 12 th - 23rd, 2021

Online - Berlin, Germany

\footnotetext{
*Presenter

${ }^{\dagger}$ The DAMPE mission is funded by the strategic priority science and technology projects in space science of Chinese Academy of Sciences. In China the data analysis is supported in part by the National Natural Science Foundation of China (Nos. U1738210, 11921003, 12003074), the Scientific Instrument Developing Project of the Chinese Academy of Sciences (No. GJJSTD20210009), the National Key Research and Development Program of China (No. 2016YFA0400200), the Strategic Priority Research Program of Chinese Academy of Sciences (No. XDB23040401), the 100 Talents Program of Chinese Academy of Sciences, the Youth Innovation Promotion Association CAS, and the Entrepreneurship and Innovation Program of Jiangsu Province. In Europe the activities and data analysis are supported by the Swiss National Science Foundation (SNSF), Switzerland, and the National Institute for Nuclear Physics (INFN), Italy.
} 


\section{INTRODUCTION}

Fermi bubbles (FBs), first discovered using the Fermi-LAT data, consist of two large bubbles, each of which is approximately $40^{\circ}$ wide and extends to $55^{\circ}$ above and below the Galactic center (GC) [1]. Since the two lobes appear to be symmetric about the Galactic plane and close to the GC when extended down to the Galactic plane, FBs are thought to be originated from the activities in the GC [1]. The average spectrum of FBs is quite hard with an index of 1.9 from $0.1 \mathrm{GeV}$ to $100 \mathrm{GeV}$ and begins to soften at around $100 \mathrm{GeV}[1,2]$. The two lobes have similar spectral parameters when fitted separately, and the intensity inside the bubbles is rather uniform [1-3] althrough a slightly harder spectrum is later found at the top of the bubbles $[4,5]$. A cocoon-like structure is detected in the southeast part which has a hard spectral index of 2.0 and is as bright as the lobe [2,6]. A jet-like feature in the FBs is also reported in [6] but is not confirmed in the later works [2,7].

The origin of FBs is still uncertain at the present time [8]. The inverse Compton(IC) interactions of high energy electrons can produce the $\gamma$-ray emission. Considering the cutoff energy at $100 \mathrm{GeV}$, the electrons should be accelerated within $\sim 10^{6} \mathrm{yr}[2]$ and the AGN jet from the GC can be a possible candidate [9]. The hadronic collision of the cosmic ray nuclei with the gas inside the bubbles can generate such emission. Since there are no constraints on the timescale of the FBs in the hadronic models, the wind or outflow from the GC could contribute these cosmic rays $[10,11]$.

The DArk Matter Particle Explorer (DAMPE) is a space-borne high energy particle telescope aiming at measuring cosmic rays and photons in a broad energy range. From the top to bottom, DAMPE is made by a plastic scintillator strip detector, a silicon-tungsten tracker-converter, a BGO imaging calorimeter and a neutron detector $[12,13]$. DAMPE can observe the $\gamma$ rays from $\sim 2 \mathrm{GeV}$ to $10 \mathrm{TeV}$ with a normal effective area of $1200 \mathrm{~cm}^{2}$ at $100 \mathrm{GeV}[13,14]$. The instrument not only has a great energy resolution which is beneficial to the search of sharp spectral structures $[13,15,16]$, but also has a strong ability to separate electrons and nuclei from the photons which may help the analyses of diffuse emission [17]. In addition, DAMPE can survey the whole sky every half year and can be useful in the study of gamma-ray sources, such as active galactic nuclei, the Crab flares and bright gamma-ray bursts $[14,18,19]$.

In this work, we use 4.8 years of DAMPE data to analyze the $\gamma$-ray emission from the FBs and provide an independent observation from the Fermi-LAT.

\section{DATA ANALYSES}

\section{$2.1 \gamma$-ray data}

We select the DAMPE flight data collected from 2016 January 1 to 2020 October 1 (DAMPE Mission Elapsed Time from 94608001 to 244512002) [17]. Events satisfying either the High Energy trigger (HET) or Low Energy trigger (LET) are chosen. We further restrict the energy from $2 \mathrm{GeV}$ and $200 \mathrm{GeV}$. The events and the corresponding time intervals when DAMPE is in the South Atlantic Anomaly (SAA) region or is strongly affected by the solar flares are removed. Both the data selection and model construction in the following are based on the dedicated DmpST package [20].

We define $5^{\circ} \leq|b| \leq 60^{\circ}$ and $|l| \leq 60^{\circ}$ as our region of interest (ROI). In the left panel of Fig. 1, the ROI binned with the CAR projection is shown along with the boundaries of the FBs. An excess of events is observed inside the bubble region. To further take into account the nonuniform 

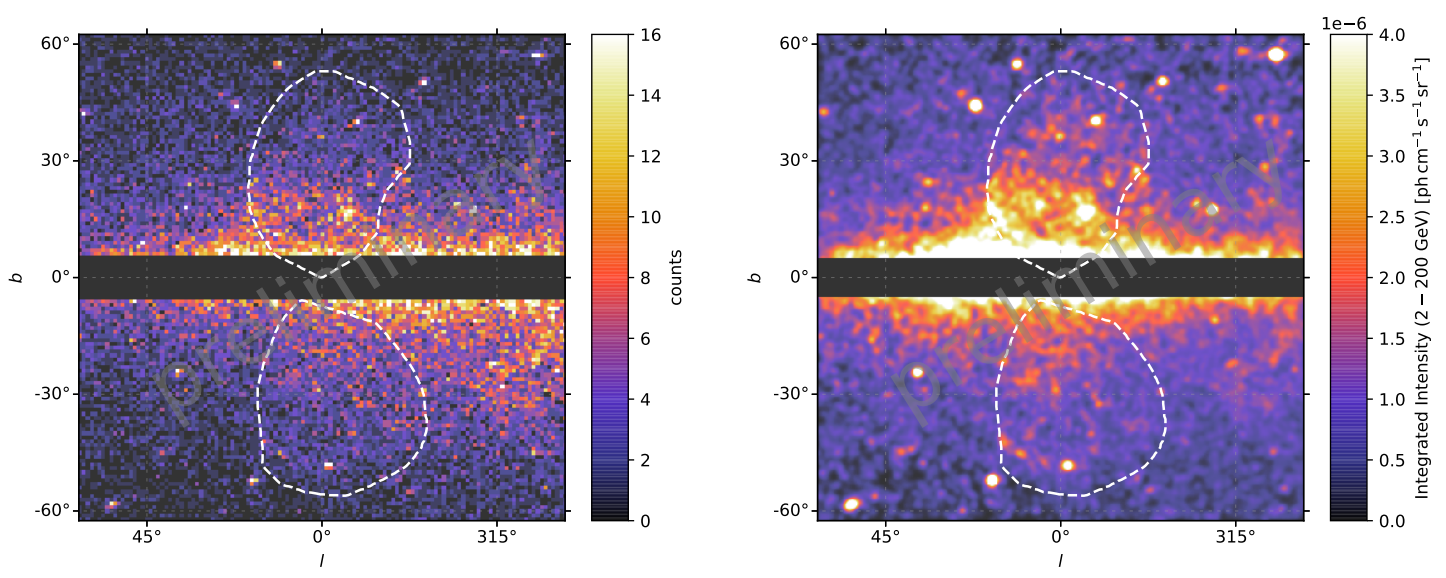

Figure 1: DAMPE counts map (left) and the integrated flux map (right) between $2 \mathrm{GeV}$ and $200 \mathrm{GeV}$ binned with the CAR projection. Emissions from the Galactic plane are removed by masking the regions with $|b|<5^{\circ}$. The shape of the lobes defined in [1] is shown in white dashed lines. The flux map is smoothed with a $0.75^{\circ}$ Gaussian kernel.

DAMPE exposure map, we also calculate the integrated intensity map as shown in the right panel of Fig. 1. We weight the exposure map with a spectral shape of $E^{-2}$ and divide the count map by the weighted exposure map to build the integrated flux map. On top of the strong diffuse emission associated with the interstellar gas, a slightly enhanced emission in the lobes and cocoon can be found.

\section{2 method and results}

We have implemented a binned likelihood analyses in this work. Firstly, the photons are binned according to the HEALPix projection [21] with nside $=64$, in which the pixel size is approximately $0.9^{\circ}$ wide. 20 logarithmically spaced energy bins are adopted to build the count cube. To reduce the influence of bright point sources, the regions within $2^{\circ}$ around the $\gamma$-ray sources in the preliminary DAMPE catalog [19] are removed from the analyses. Then we construct the $\gamma$-ray model by using the Galactic diffuse emission (GDE) model gll_iem_v02 . fit,${ }^{1}$ the isotropic extragalactic emission model, and the flat FBs template [1]. We set the LogParabola and PowerLaw spectral shapes for the GDE and isotropic emission respectively. All the maps will be multiplied by the exposure cube and then folded with the point-spread function [22]. Finally, the best-fit spectral parameters can be achieved by optimizing the likelihood function using the MINUIT algorithm [23].

As a starting points, we have built a residual map when the FBs template is not included in the model. The map in the left panel of Fig. 2 clearly shows an excess with respect to the diffuse emission, which is localized in the lobes of the FBs. To check whether the excess is related to other diffuse emission, we also plot the countours of HI gas [24] and the Loop I [25] in the right panel. Neither of them can match the shape of the excess.

We first analyze the two lobes of FBs as a whole. If we choose the PowerLaw spectrum for the FBs, their TS value is 330.8 which corresponds to a significance of $18.0 \sigma$ for a $\chi^{2}$

\footnotetext{
1https://fermi.gsfc.nasa.gov/ssc/data/access/lat/ring_for_FSSC_final4.pdf
} 

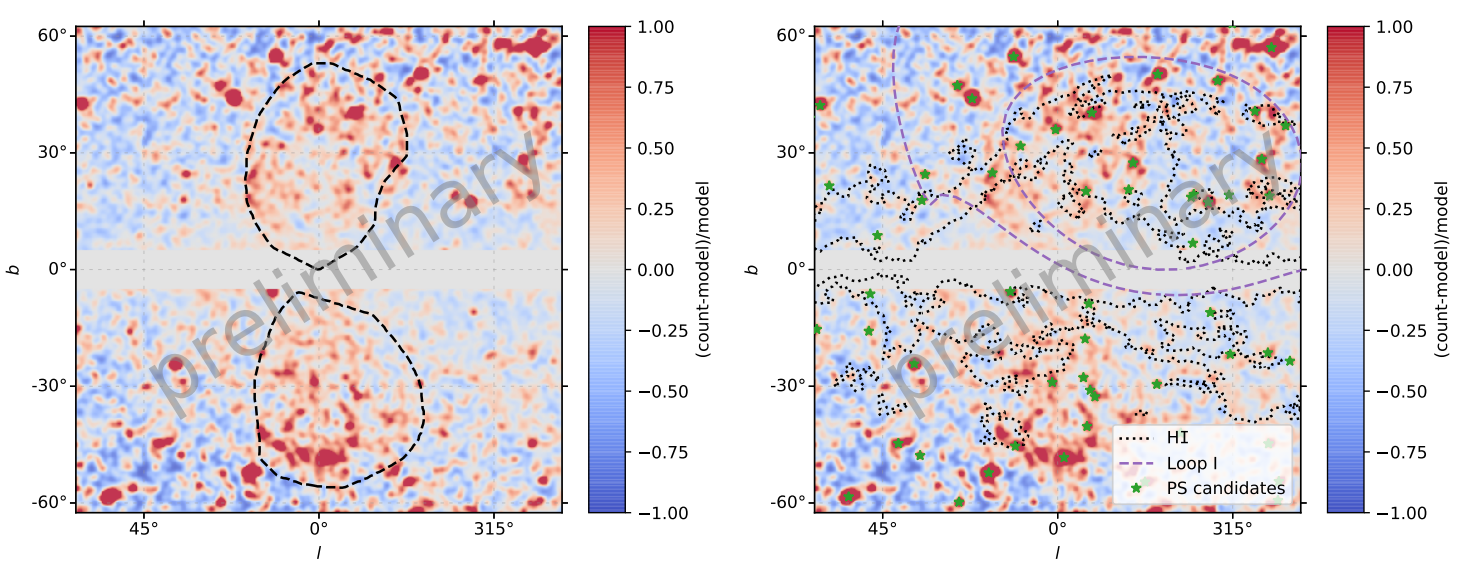

Figure 2: Residual map when FBs are not included in the model. The map is smoothed with a $0.75^{\circ}$ Gaussian kernel to reduce the fluctuation. Both the panels show the same map but with different contours overlaid. The black dashed contours in the left panel correspond to the edges of lobes [1]. The black dotted and purple dashed lines in the right panel show the contours of HI gas [24] and Loop I [25]. Green stars represent the 5-yr point source candidates [19].

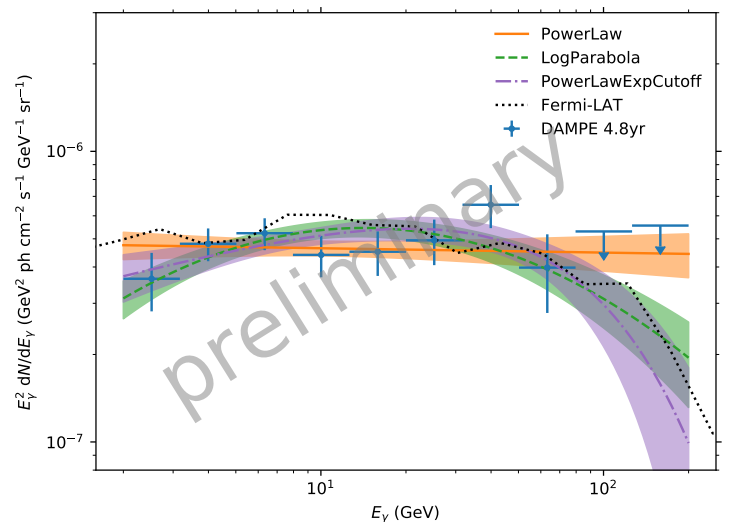

Figure 3: Average spectral energy distributions (SEDs) of FBs. The color bands correspond to the $1 \sigma$ statistical uncertainties of models after global fittings. The black dashed line shows the SED from [2]. Upper limits are presented when the TS value is $<10$.

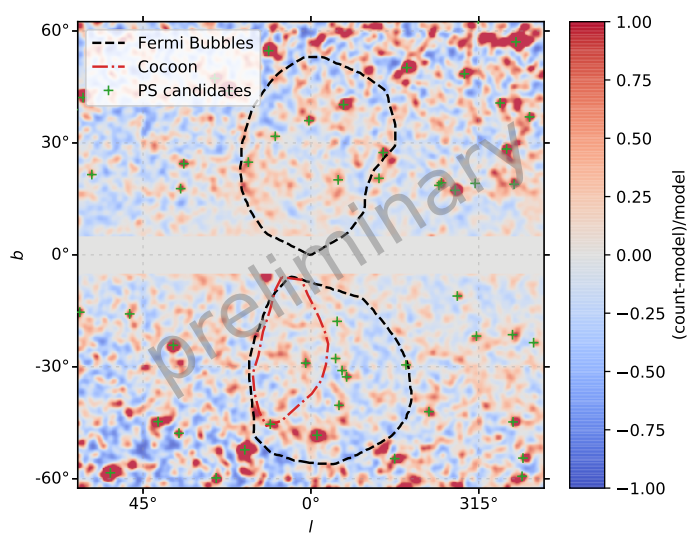

Figure 4: Residual map when FBs are included in the model. A $0.75^{\circ}$ Gaussian kernel is used to smooth the map. The black dashed and red dotdashed lines represent the edges of two lobes [1] and the cocoon [6].

distribution with 2 degrees of freedom [26]. The spectral index is found to be $-2.01 \pm 0.05$ and the integrated intensity from $2 \mathrm{GeV}$ to $200 \mathrm{GeV}$ is $F_{2-200}=(2.3 \pm 0.2) \times 10^{-7} \mathrm{ph} \mathrm{cm}^{-2} \mathrm{~s}^{-1} \mathrm{sr}^{-1}$. The predicted DAMPE photon count originated from the FBs is around 1700 for the best-fit model. We also test several other spectral shapes. For the LogParabola spectrum, the TS value becomes 339.1 which means that the significance of FBs is $18.1 \sigma$ and that the significance of this spectral model with respect to the simple power law model is $2.9 \sigma$. The best-fit spectral parameters are $F_{2-200}=(2.1 \pm 0.2) \times 10^{-7} \mathrm{ph} \mathrm{cm}^{-2} \mathrm{~s}^{-1} \mathrm{sr}^{-1}, \alpha=1.5 \pm 0.1$ and $\beta=0.14 \pm 0.04$. For the 

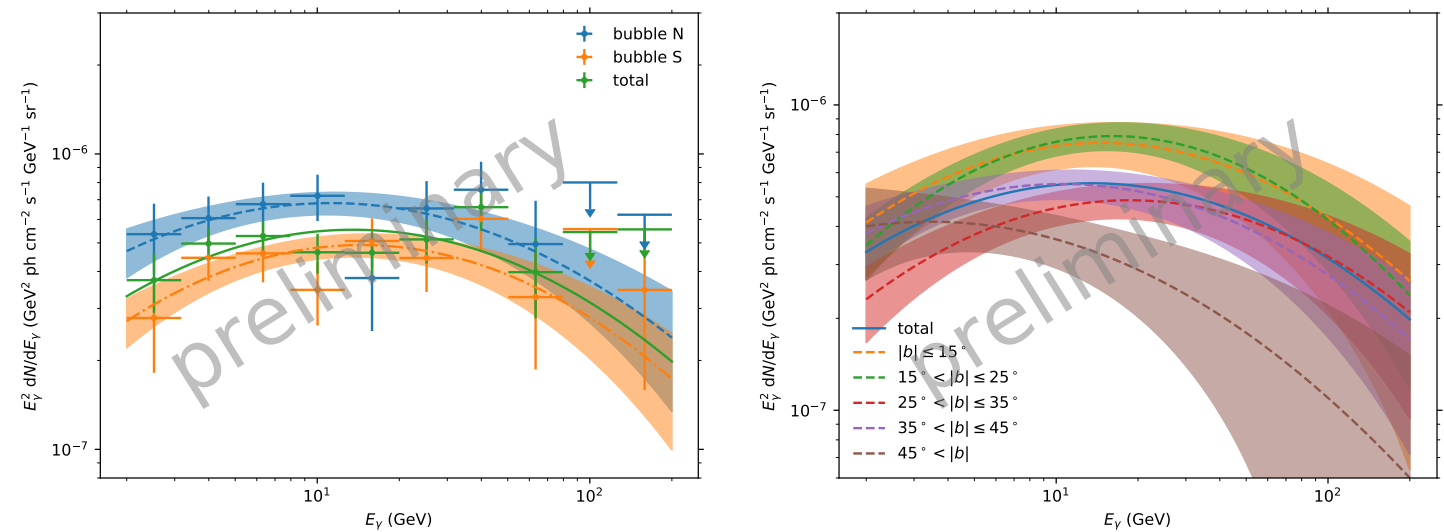

Figure 5: SEDs of the different regions of the FBs. The left panel shows the SEDs from the north (blue) and south (orange) lobes. The right panel presents the SEDs from several latitude slices of FBs. The lines and bands are the best-fit LogParabola models and their $1 \sigma$ uncertainties respectively.

PowelawExpCutoff spectrum, the TS value is 339.5 for the FBs. The spectral index and cutoff energy are $-1.7 \pm 0.2$ and $78 \pm 40 \mathrm{GeV}$ respectively. All the fitted models and their uncertainties are given in Fig. 3 along with the spectral energy distributions (SEDs). The results are consistent with those from the Fermi-LAT [2]. The residual map when the best-fit LogParabola model is included in the model is shown in Fig. 4. Since the fluctuations inside and outside the lobes are almost the same, the excess above and below the GC is well extracted.

In this work we have also analyzed the different regions of the FBs separately. We use the LogParabola spectral types since it is more favored than the PowerLaw spectrum and is comparable to the PowerlawExpCutoff model. We first divide the FBs into the north and south lobes and analyze them separately. The TS values (significance) for the north and south bubbles are $193.7(13.5 \sigma)$ and $194.6(13.6 \sigma)$ respectively. The obtained spectra are shown in the left panel of Fig. 5. The intensity of the north lobe is stronger than the south one in the low energy range, which is probably influenced by the GDE emission that is stronger in the northern sky. Then we split the FBs template into different latitude slices and calculate their spectra as shown in the right panel of Fig. 5. The TS values (significance) are $49.1(6.4 \sigma), 142.5(11.5 \sigma), 88.3(8.9 \sigma), 129.4(10.9 \sigma)$, and $41.2(5.8 \sigma)$ for the slices from low to high latitude. They generally exhibit a hard spectrum similar to that of the whole FB template, although the lower latitude parts tend to be stronger and harder than the higher latitude parts.

Since more positive residuals appear in the southeast than in the southweast in Fig. 4 which may correlate to the emission from the cocoon, we also search for its emission. We include the cocoon template from the Fig. 2 of [6] with a PowerLaw spectral shape in the model. The TS value for the cocoon is only 11.7 , corresponding to a significance of $3.0 \sigma$. Since the structure is not significantly detected, the $95 \%$ confidence level upper limit on the spectrum is presented in Fig. 6 assuming the spectral index to be 2.0. The cocoon intensity from the Fermi-LAT [2] is also shown in the figure, and our result is consistent with the previous result. 


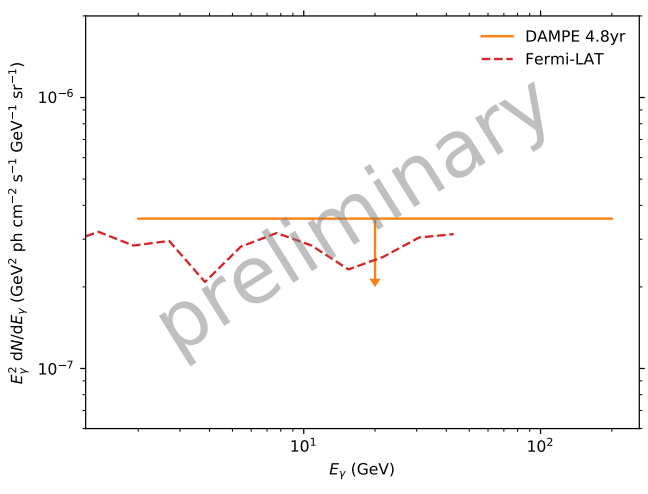

Figure 6: $95 \%$ confidence level upper limit on the spectrum of the cocoon. The spectral index of the cocoon is assumed to be 2.0. The red dashed line represent the spectrum from [2].

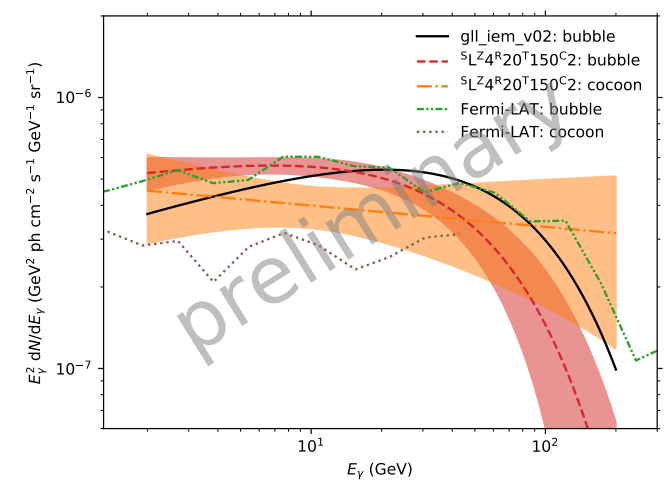

Figure 7: Spectra of the FBs and cocoon derived with the alternative Galactic diffuse emission (GDE) model [22]. The black solid line is the result using the baseline GDE model. The green and purple lines correspond to the spectra of FBs and cocoon in [2].

\section{3 systematic uncertainty}

Since GDE contributes to the most emission in the ROI, its model will significantly influence the spectra of the FBs and cocoon. The model gll_iem_v02. fit we adopted as the baseline is built by fitting the $\gamma$-ray data with the interstellar gas column densities and the IC model from the Galactic cosmic ray. However it can not well describe some of the regions above the GC and in the Galactic plane [27] and may absorb the emission from the excess and thereby change the spectra and morphologies of the target sources. For this reason, we have repeated the analyses with an alternative GDE model.

The Galprop models are commonly adopted to evaluate the uncertainties resulted from the GDE emission [22, 28]. We change the baseline GDE model with the pion decay, IC and bremsstrahlung templates calculated with the parameter set ${ }^{\mathrm{S}} \mathrm{L}^{\mathrm{Z}} 4^{\mathrm{R}} 20^{\mathrm{T}} 150^{\mathrm{C}} 2$. The Loop I template from [25] is also included in the model. We set free the spectral shapes of the pion decay component, the isotropic emission, Loop I, FBs and cocoon, and keep fixed the remaining components in the fits.

Fig. 7 shows the derived spectra from the FBs and cocoon. The TS value and corresponding significance for the lobes are 281.4 and $16.3 \sigma$ respectively if the PowerlawExpCutoff spectral shape is chosen. The best-fit spectral index and cutoff energy are $-1.9 \pm 0.1$ and $55 \pm 29 \mathrm{GeV}$. On the other hand, a TS value is 33.2 for the cocoon which equals to a $5.4 \sigma$ significance. The intensity and index are $(2.1 \pm 0.5) \times 10^{-7} \mathrm{ph} \mathrm{cm}^{-2} \mathrm{~s}^{-1} \mathrm{sr}^{-1}$ and $-2.1 \pm 0.2$ respectively. The flux is higher than that of Fermi-LAT even when the statistical uncertainties are involved.

\section{SUMMARY}

FBs are the new diffuse structure discovered by Fermi-LAT and thought to be related to the activities from the GC. In this work, we use 4.8-yr DAMPE photon data to analyze their emission. The significance of the FBs as a whole is found to be $18.0 \sigma$ if the standard GDE model is adopted. The spectrum is well consistent with that from the Fermi-LAT [2]. The data indicate a mild 
curvature in spectrum at a significance of $2.9 \sigma$. If fitted separately, the north and south lobes still have significances of $13.5 \sigma$ and $13.6 \sigma$ respectively. A weak excess is also suggested in the cocoon region and its significance is around 3.0 $\sigma$. The uncertainty from the GDE model is also shown by using an alternative model, which has a great influence on the cutoff energy of the FBs and normalization of the cocoon. In the future, we will implement a GDE model with the DAMPE data and optimize the analyses.

\section{References}

[1] M. Su, T.R. Slatyer and D.P. Finkbeiner, Giant Gamma-ray Bubbles from Fermi-LAT: Active Galactic Nucleus Activity or Bipolar Galactic Wind?, Astrophys. J. 724 (2010) 1044 [1005 . 5480].

[2] Fermi-LAT collaboration, The Spectrum and Morphology of the Fermi Bubbles, Astrophys. J. 793 (2014) 64 [1407.7905].

[3] D. Hooper and T.R. Slatyer, Two emission mechanisms in the Fermi Bubbles: A possible signal of annihilating dark matter, Physics of the Dark Universe 2 (2013) 118 [1302 . 6589].

[4] R.-Z. Yang, F. Aharonian and R. Crocker, The Fermi bubbles revisited, Astron. Astrophys. 567 (2014) A19 [1402.0403].

[5] S.A. Narayanan and T.R. Slatyer, A latitude-dependent analysis of the leptonic hypothesis for the Fermi Bubbles, Mon. Not. R. Astron. Soc. 468 (2017) 3051 [1603. 06582].

[6] M. Su and D.P. Finkbeiner, Evidence for Gamma-Ray Jets in the Milky Way, Astrophys. J. 753 (2012) 61 [1205.5852].

[7] M. Selig, V. Vacca, N. Oppermann and T.A. Enßlin, The denoised, deconvolved, and decomposed Fermi $\gamma$-ray sky. An application of the $D^{3}$ PO algorithm, Astron. Astrophys. 581 (2015) A126 [1410.4562].

[8] H.Y. Yang, M. Ruszkowski and E. Zweibel, Unveiling the Origin of the Fermi Bubbles, Galaxies 6 (2018) 29 [1802.03890].

[9] F. Guo and W.G. Mathews, The Fermi Bubbles. I. Possible Evidence for Recent AGN Jet Activity in the Galaxy, Astrophys. J. 756 (2012) 181 [1103.0055].

[10] R.M. Crocker and F. Aharonian, Fermi Bubbles: Giant, Multibillion-Year-Old Reservoirs of Galactic Center Cosmic Rays, Phys. Rev. Lett. 106 (2011) 101102 [1008 . 2658].

[11] G. Mou, F. Yuan, D. Bu, M. Sun and M. Su, Fermi Bubbles Inflated by Winds Launched from the Hot Accretion Flow in Sgr A*, Astrophys. J. 790 (2014) 109 [1403.2129].

[12] J. Chang, Dark Matter Particle Explorer: The First Chinese Cosmic Ray and Hard $\gamma$-ray Detector in Space, Chin. J. Spac. Sci. 34 (2014) 550.

[13] DAMPE collaboration, The DArk Matter Particle Explorer mission, Astropart. Phys. 95 (2017) 6 [1706.08453].

[14] X. Li, K.-K. Duan, W. Jiang, Z.-Q. Shen and M.M. Salinas, Recent $\gamma$-ray Results from DAMPE, in proceedings of ICRC2019, PoS (ICRC2019) 576 (2019). 
[15] Z.-Q. Shen, Y.-F. Liang, K.-K. Duan and Y.-Z. Fan, Search for a gamma-ray line feature with DAMPE, in proceedings of ICRC2019, PoS (ICRC2019) 601 (2019).

[16] Z.-L. Xu, K.-K. Duan, X. Li, M.N. Mazziotta and Z.-Q. Shen, Search for gamma-ray lines in the Galaxy with DAMPE, in proceedings of ICRC2021, PoS (ICRC2021) 632 (2021).

[17] Z.-L. Xu, K.-K. Duan, Z.-Q. Shen, S.-J. Lei et al., An algorithm to resolve $\gamma$-rays from charged cosmic rays with DAMPE, Res. Astron. Astrophys. 18 (2018) 027 [1712. 02939].

[18] S.-J. Lei, Q. Yuan, Z.-L. Xu, K.-K. Duan and M. Su, Gamma-ray Astronomy with DAMPE, in proceedings of ICRC2017, PoS (ICRC2017) 616 (2017).

[19] K.-K. Duan, W. Jiang, X. Li, Z.-Q. Shen and Z.-L. Xu, Observations of gamma-ray sources with DAMPE, in proceedings of ICRC2021, PoS (ICRC2021)631 (2021).

[20] K.-K. Duan, W. Jiang, Y.-F. Liang, Z.-Q. Shen et al., DmpIRFs and DmpST: DAMPE instrument response functions and science tools for gamma-ray data analysis, Res. Astron. Astrophys. 19 (2019) 132 [1904 . 13098].

[21] K.M. Górski, E. Hivon, A.J. Banday, B.D. Wandelt et al., HEALPix: A Framework for High-Resolution Discretization and Fast Analysis of Data Distributed on the Sphere, Astrophys. J. 622 (2005) 759 [astro-ph/0409513].

[22] FERMI-LAT collaboration, Fermi-LAT Observations of the Diffuse $\gamma$-Ray Emission: Implications for Cosmic Rays and the Interstellar Medium, Astrophys. J. 750 (2012) 3 [1202 . 4039].

[23] F. James and M. Roos, Minuit - a system for function minimization and analysis of the parameter errors and correlations, Comput. Phys. Commun. 10 (1975) 343.

[24] N. Ben Bekhti, L. Flöer, R. Keller, J. Kerp et al., HI4PI: A full-sky H I survey based on EBHIS and GASS, Astron. Astrophys. 594 (2016) A116 [1610.06175].

[25] M. Wolleben, A New Model for the Loop I (North Polar Spur) Region, Astrophys. J. 664 (2007) 349 [0704.0276].

[26] S.S. Wilks, The large-sample distribution of the likelihood ratio for testing composite hypotheses, Ann. Math. Statist. 9 (1938) 60.

[27] FERMI-LAT collaboration, Development of the Model of Galactic Interstellar Emission for Standard Point-source Analysis of Fermi Large Area Telescope Data, Astrophys. J. Suppl. Ser. 223 (2016) 26 [1602.07246].

[28] F. de Palma, T.J. Brandt, G. Johannesson and L. Tibaldo, A Method for Exploring Systematics Due to Galactic Interstellar Emission Modeling: Application to the Fermi LAT SNR Catalog, arXiv:1304 . 1395 (2013). 


\section{Full Authors List: DAMPE Collaboration}

F. Alemanno ${ }^{1,2}$, Q. An ${ }^{3,4}$, P. Azzarello ${ }^{5}$, F. C. T. Barbato ${ }^{1,2}$, P. Bernardini ${ }^{6,7}$, X. J. Bi ${ }^{8,9}$, M. S. Cai ${ }^{10,11}$, E. Casilli6, ${ }^{6,7}$, E. Catanzani ${ }^{12}$, J. Chang ${ }^{10,11}$, D. Y. Chen ${ }^{9,10}$, J. L. Chen ${ }^{13}$, Z. F. Chen ${ }^{10,11}$, M. Y. Cui ${ }^{10}$, T. S. Cui ${ }^{14}$, Y. X. Cui ${ }^{10,11}$, H. T. Dai ${ }^{3,4}$, A. De Benedittis ${ }^{6,7}$,

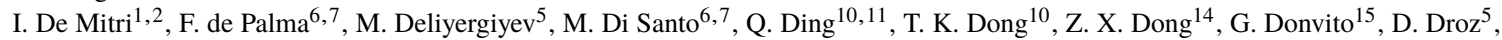
J. L. Duan ${ }^{13}$, K. K. Duan ${ }^{10}$, D. D’Urso ${ }^{12, *}$, R. R. Fan ${ }^{8}$, Y. Z. Fan ${ }^{10,11}$, K. Fang ${ }^{8}$, F. Fang ${ }^{13}$, C. Q. Feng ${ }^{3,4}$, L. Feng ${ }^{10}$, P. Fusco ${ }^{15,16}$,

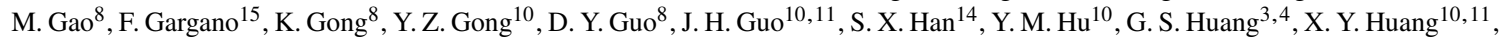

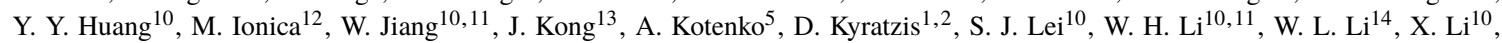

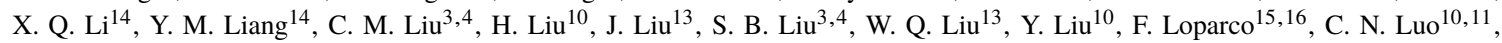
M. $\mathrm{Ma}^{14}$, P. X. $\mathrm{Ma}^{10}$, T. $\mathrm{Ma}^{10}$, X. Y. Ma ${ }^{14}$, G. Marsella $a^{6,7, \dagger}$, M. N. Mazziotta ${ }^{15}$, D. Mo ${ }^{13}$, X. Y. $\mathrm{Niu}^{13}$, X. Pan ${ }^{10,11}$, A. Parenti ${ }^{1,2}$, W. X. Peng ${ }^{8}$, X. Y. Peng ${ }^{10}$, C. Perrina ${ }^{5, \ddagger}$, R. Qiao ${ }^{8}$, J. N. Rao ${ }^{14}$, A. Ruina ${ }^{5}$, M. M. Salinas ${ }^{5}$, G. Z. Shang ${ }^{14}$, W. H. Shen ${ }^{14}$, Z. Q. Shen ${ }^{10}$, Z. T. Shen ${ }^{3,4}$, L. Silveri ${ }^{1,2}$, J. X. Song ${ }^{14}$, M. Stolpovskiy ${ }^{5}, \mathrm{H} \mathrm{Su}^{13}$, M. Su${ }^{17}$, Z. Y. Sun ${ }^{13}$, A. Surdo ${ }^{7}$, X. J. Teng ${ }^{14}$, A. Tykhonov ${ }^{5}$, H. Wang ${ }^{14}$, J. Z. Wang ${ }^{8}$, L. G. Wang ${ }^{14}$, S. Wang ${ }^{10,11}$, S. X. Wang ${ }^{10,11}$, X. L. Wang ${ }^{3,4}$, Y. Wang ${ }^{3,4}$, Y. F. Wang $g^{3,4}$, Y. Z. Wang ${ }^{10}$, D. M. $\mathrm{Wei}^{10,11}$, J. J. Wei ${ }^{10}$, Y. F. Wei ${ }^{3,4}$, D. $\mathrm{Wu}^{8}$, J. Wu ${ }^{10,11}$, L. B. Wu ${ }^{1,2,3,4}$, S. S. Wu ${ }^{14}$ X. Wu ${ }^{5}$ Z. Q. Xia ${ }^{10}$, E. H. Xu ${ }^{3,4}$, H. T. $\mathrm{Xu}^{14}$, Z. H. Xu ${ }^{10,11}$, Z. L. Xu ${ }^{10}$, Z. Z. Xu ${ }^{3,4}$, G. F. Xue ${ }^{14}$, H. B. Yang ${ }^{13}$, P. Yang ${ }^{13}$, Y. Q. Yang ${ }^{13}$, H. J. Yao ${ }^{13}$, Y. H. Yu ${ }^{13}$, G. W. Yuan ${ }^{10,11}$, Q. Yuan ${ }^{10,11}$, C. Yue ${ }^{10}$, J. J. Zang ${ }^{10, \text { II }}$, F. Zhang ${ }^{8}$, S. X. Zhang ${ }^{13}$, W. Z. Zhang ${ }^{14}$, Y. Zhang ${ }^{10}$, Y. J. Zhang ${ }^{13}$, Y. L. Zhang ${ }^{3,4}$, Y. P. Zhang ${ }^{13}$, Y. Q. Zhang ${ }^{10}$, Z. Zhang ${ }^{10}$, Z. Y. Zhang ${ }^{3,4}$, C. Zhao ${ }^{3,4}$, H. Y. Zhao ${ }^{13}$, X. F. Zhao ${ }^{14}$, C. Y. Zhou ${ }^{14}$, and Y. Zhu ${ }^{14}$

${ }^{1}$ Gran Sasso Science Institute (GSSI), Via Iacobucci 2, I-67100 L'Aquila, Italy. ${ }^{2}$ Istituto Nazionale di Fisica Nucleare (INFN) -Laboratori Nazionali del Gran Sasso, I-67100 Assergi, L’Aquila, Italy. ${ }^{3}$ State Key Laboratory of Particle Detection and Electronics, University of Science and Technology of China, Hefei 230026, China. ${ }^{4}$ Department of Modern Physics, University of Science and Technology of China, Hefei 230026, China. ${ }^{5}$ Department of Nuclear and Particle Physics, University of Geneva, CH-1211, Switzerland. ${ }^{6}$ Dipartimento di Matematica e Fisica E. De Giorgi, Università del Salento, I-73100, Lecce, Italy. ${ }^{7}$ Istituto Nazionale di Fisica Nucleare (INFN) Sezione di Lecce, I-73100, Lecce, Italy. ${ }^{8}$ Institute of High Energy Physics, Chinese Academy of Sciences, Yuquan Road 19B, Beijing 100049, China. ${ }^{9}$ University of Chinese Academy of Sciences, Yuquan Road 19A, Beijing 100049, China. ${ }^{10}$ Key Laboratory of Dark Matter and Space Astronomy, Purple Mountain Observatory, Chinese Academy of Sciences, Nanjing 210023, China. ${ }^{11}$ School of Astronomy and Space Science, University of Science and Technology of China, Hefei 230026, China. ${ }^{12}$ Istituto Nazionale di Fisica Nucleare (INFN) - Sezione di Perugia, I-06123 Perugia, Italy. ${ }^{13}$ Institute of Modern Physics, Chinese Academy of Sciences, Nanchang Road 509, Lanzhou 730000, China. ${ }^{14}$ National Space Science Center, Chinese Academy of Sciences, Nanertiao 1, Zhongguancun, Haidian district, Beijing 100190, China. ${ }^{15}$ Istituto Nazionale di Fisica Nucleare (INFN) - Sezione di Bari, I-70125, Bari, Italy. ${ }^{16}$ Dipartimento di Fisica "M. Merlin" dell’Università e del Politecnico di Bari, I-70126, Bari, Italy. ${ }^{17}$ Department of Physics and Laboratory for Space Research, the University of Hong Kong, Pok Fu Lam, Hong Kong SAR, China. *Now at Università di Sassari, Dipartimento di Chimica e Farmacia, I-07100, Sassari, Italy. ${ }^{\dagger}$ Now at Università degli Studi di Palermo, Dipartimento di Fisica e Chimica "E. Segrè", via delle Scienze ed. 17, I-90128 Palermo, Italy. ${ }^{\ddagger}$ Also at Institute of Physics, Ecole Polytechnique Federale de Lausanne (EPFL), CH-1015 Lausanne, Switzerland. ${ }^{\S}$ Now at Shandong Institute of Advanced Technology (SDIAT), Jinan, Shandong, 250100, China. II Also at School of Physics and Electronic Engineering, Linyi University, Linyi 276000, China. 